Cahiers « Mondes anciens »

ANCIENS

Histoire et anthropologie des mondes anciens

5 | 2014

Maudire et mal dire : paroles menaçantes en Grèce ancienne

\title{
Théomnestos au tribunal ou l'injure comme arme du citoyen
}

Francis Larran

\section{CpenEdition}

Journals

Édition électronique

URL : http://journals.openedition.org/mondesanciens/1241

DOI : 10.4000/mondesanciens. 1241

ISSN : 2107-0199

Éditeur

UMR 8210 Anthropologie et Histoire des Mondes Antiques

Référence électronique

Francis Larran, "Théomnestos au tribunal ou l'injure comme arme du citoyen », Cahiers " Mondes anciens » [En ligne], 5 | 2014, mis en ligne le 14 février 2014, consulté le 01 mai 2019. URL : http:// journals.openedition.org/mondesanciens/1241; DOI : 10.4000/mondesanciens.1241

Ce document a été généré automatiquement le 1 mai 2019.

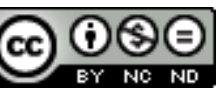

Les Cahiers «Mondes Anciens » sont mis à disposition selon les termes de la licence Creative Commons Attribution - Pas d'Utilisation Commerciale - Pas de Modification 4.0 International. 


\title{
Théomnestos au tribunal ou l'injure comme arme du citoyen
}

\author{
Francis Larran
}

1 Ah! Si seulement Théomnestos l'avait traité de «meurtrier » (androphonos)! La diffamation précise et franche aurait probablement simplifié la tâche du client anonyme pour lequel Lysias a composé le Contre Théomnestos. L'accusation à l'origine d'un procès ouvert en 384-383 avant J.-C. n'en aurait été que mieux étayée, les plaidoiries sans doute moins longues et le verdict des jurés certainement favorable au plaignant. Tels n'ont pourtant pas été les mots de Théomnestos.

2 Traîné en justice, quelque temps auparavant, par un certain Lysithéos qui lui reprochait d'avoir parlé devant le peuple alors que, ayant jeté son bouclier au front, il tombait sous le coup d'une interdiction légale, Théomnestos aurait alors prétendu que le client de Lysias, appelé comme témoin à charge contre lui, avait tué son propre père ${ }^{1}$. L'attaque était violente, mais la formule bien vague. Simple hasard ou habile stratégie visant à déstabiliser le témoin de Lysithéos? La question ne fait pas de doute pour le client de Lysias : au cours du procès intenté par Lysithéos, Théomnestos a fait allusion, même s'il s'en défend ensuite ${ }^{2}$, à un mot injurieux interdit par la loi. Le plaignant décide dès lors d'intenter contre son accusateur une action privée assez peu employée ${ }^{3}$ : la kakègorias dikè (l'action d'« injure »)

3 Dans l'Athènes classique, l'injure verbale n'est pas punissable en règle générale ${ }^{5}$. Pour qu'elle soit traitée en délit, trois conditions sont requises : l'injure doit s'adresser aux morts ; elle doit être proférée dans un certain nombre de lieux (sanctuaires, tribunaux, locaux occupés par un magistrat, lors de l'assistance aux jeux $)^{6}$; elle doit utiliser un des termes interdits (aporrhèta). Théomnestos semble avoir enfreint deux lois: la loi solonienne car il a injurié son adversaire «au cours du procès $»^{7}$ et pourrait pour cette raison se voir intenter une action publique; la loi sur les aporrhèta car il a traité de parricide le client de Lysias.

4 C'est uniquement pour cette dernière faute que Théomnestos est poursuivi ${ }^{8}$ et encourt une lourde amende de cinq cents drachmes'. En effet, prononcer un des mots interdits ( aporrhèta $)^{10}$ porte atteinte à la moralité familiale et à la société : la loi interdit notamment 
d'insulter quelqu'un en le traitant de meurtrier (androphonos), en prétendant qu'il bat son père ou sa mère (patraloias, mètraloias), en stipulant qu'il a abandonné son bouclier sur le champ de bataille ${ }^{11}$, ou encore en le stigmatisant pour ses activités de boutiquier exercées, sur l'agora ${ }^{12}$.

Que faut-il voir dans la querelle de mots qui occupe tout le Contre Théomnestos de Lysias? Une affaire bien mince qui, pour opposer un plaignant tatillon à un accusé opportuniste capable de jouer sur les termes, ne peut avoir retenu l'attention de Lysias, comme le supposent quelques érudits contemporains qui mettent en doute la paternité du discours ${ }^{13}$ ? Un simple fait divers judiciaire dont les subtilités techniques ne sont susceptibles d'intéresser que les spécialistes du droit athénien? Une pièce utile dans les débats historiographiques sur la date de création de la loi relative aux aporrhèta ${ }^{14}$ ? Si le procès de 384-383 ne joue assurément pas un rôle majeur dans l'histoire athénienne, il a cependant retenu toute l'attention de Louis Gernet qui voit dans les aporrhèta des injures néfastes à même, par leur seule énonciation, de souiller le groupe ${ }^{15}$. L'affaire du Contre Théomnestos ouvre également la voie à qui tente d'éclairer le droit athénien en le mettant en relation avec la société et le milieu culturel de l'époque.

Un travail de recontextualisation historique des injures évoquées dans le Contre Théomnestos est indispensable si l'on souhaite éviter le plus grave des écueils: l'anachronisme. En effet, les injures ne doivent pas être considérées comme des objets intemporels susceptibles de traverser, immuables, les siècles. Elles ont, elles aussi, une histoire, au cours de laquelle leur sens comme leur gravité fluctuent. La sensibilité des Athéniens à l'égard des termes aporrhèta semble notamment plus vive au début du $\mathrm{IV}^{\mathrm{e}}$ siècle qu'au siècle précédent ${ }^{16}$. Si les termes patraloias et mètraloias sont attestés dans les sources du ve siècle, il n'est jamais question de condamner ceux qui ont choisi de les prononcer ${ }^{17}$, alors même que leur énonciation constitue un délit évident aux yeux du client de Lysias ${ }^{18}$. Dans une perspective comparable, la spécificité historique des aporrhèta s'apprécie, au IV ${ }^{e}$ siècle, à l'aune de la récurrence d'autres injures qui sont tolérées dans les plaidoyers des orateurs attiques, alors même qu'elles semblent, elles aussi, porter atteinte à l'ordre social. Aussi violentes soient-elles, les injures de "prostitué " $^{19}$, « voleur », «Scythe » ou « corrompu $»^{20}$ restent moins graves qu'androphonos ou patraloias, car elles ne sont ni sacrilèges ni interdites par une loi qui s'est constituée progressivement à partir du $\mathrm{VI}^{\mathrm{e}}$ siècle (Glotz 1900, p. 790). C'est sans doute ces deux caractéristiques qui expliquent l'intérêt des contemporains de Théomnestos pour les aporrhèta.

7 Dans l'Athènes du début du IV siècle encore traumatisée par la Guerre du Péloponnèse, qui a sans doute donné libre cours au déchaînement des passions et, à la suite de la peste, à divers manquements à l'ordre moral et "religieux ", une guerre qui a fait vaciller les valeurs traditionnelles sous les coups de boutoir de l'amoralisme politique et de l'utilisation opportuniste des thèses sophistiques, un conflit qui a déstabilisé le régime démocratique en portant par deux fois au pouvoir des oligarques (411 et 404-403) et qui a enfin plongé le corps civique dans une stasis sanglante et brutale, les regards se font plus attentifs au respect des normes juridiques et religieuses et se tournent désormais vers le passé, la "constitution des ancêtres " comme les lois archaïques dont la capacité à traverser les siècles suscite l'admiration (Romilly 1971, p. 203-225). C'est bien à cette époque que Solon, qui a notamment cherché à purifier la parole publique en interdisant d'insulter un particulier au tribunal ${ }^{21}$, obtient le statut de législateur mythique. C'est à lui qu'il faut se référer pour espérer reconstruire une cité stable au régime démocratique 
modéré (Mossé 1979, p. 425-437) et ainsi se placer à l'abri de procès irresponsables qui ont pu condamner des innocents tels que le sage Socrate ${ }^{22}$. Paroles agressives, paroles impies, les aporrhèta sont aussi des termes condamnés par une loi héritée du passé, dont l'esprit est censé guider Athènes sur la voie pieuse de la restauration et de la pacification. Les prononcer au début $\mathrm{du} \mathrm{IV}^{\mathrm{e}}$ siècle, c'est en quelque sorte faire montre d'incivisme en réintroduisant le désordre dans une cité qui a précisément comme ambition d'oublier les maux de la Guerre du Péloponnèse pour mieux se reconstruire sur la base d'un passé idéalisé (Loraux 1997a).

8 Ainsi recontextualisées, les stratégies développées par les deux parties lors du procès de 384-383 ouvrent une fenêtre sur les débats qui agitent Athènes au début $\mathrm{du} \mathrm{IV}^{\mathrm{e}}$ siècle tout comme sur le rôle politique de l'Héliée. Scène démocratique où chaque plaideur se présente comme un citoyen exemplaire pour mieux reprocher à son adversaire les pires vices civiques, le tribunal est un lieu de médiation politique dans lequel transitent, par le biais des plaidoiries, les principaux topoi démocratiques nourris à la source des débats intellectuels. Prononcer, interpréter, condamner, utiliser, manipuler l'injure au tribunal sont autant d'actions qui engagent pleinement l'accusé comme son adversaire dans leur rapport à la cité. Jouer au bon citoyen, participer à l'agôn oral judiciaire, prendre position à l'égard des aporrhèta, c'est choisir de se livrer à une sorte de dokimasie au cours de laquelle les jurés interrogent le comportement, le rapport au langage et même à l'histoire de chaque plaideur. Telle semble être en tout cas l'épreuve imposée à Théomnestos et à son adversaire en 384-383.

\section{L'injure, une arme judiciaire}

Qu'elle soit lancée par Théomnestos ou condamnée par le client de Lysias, l'injure constitue, pour les deux acteurs du procès, la pièce maîtresse de leur stratégie judiciaire. À chaque fois, il s'agit de montrer combien l'adversaire bafoue, par son impiété et ses mœurs violentes, par sa parole transgressive, les normes d'une morale civique prompte à condamner les excès comportementaux en raison de la menace qu'ils font peser sur la cohésion de la cité.

La colère seule peut-elle être à l'origine de l'insulte abominable lancée par Théomnestos contre le client de Lysias lors du procès intenté par Lysithéos? L'argument, utilisé par Théomnestos ${ }^{23}$, est sans doute trop simple. Accuser son adversaire de parricide, c'est-àdire d'un crime monstrueux ${ }^{24}$ et déshonorant ${ }^{25}$, possède en effet bien des vertus judiciaires, car les meurtriers ne peuvent, d'après le droit athénien, prendre la parole en public $^{26}$. La manœuvre est habituelle ${ }^{27}$. Lui-même victime d'un tel procédéé ${ }^{28}$, Théomnestos cherche aussi probablement à rappeler aux jurés du procès de Lysithéos que le client de Lysias tombe sous le coup de la loi qui interdit aux enfants qui maltraitent leurs parents de prendre la parole à la tribune ${ }^{29}$ et ne peut, par conséquent, se présenter en tant que témoin à charge.

11 Outre la souillure que le parricide pourrait communiquer à la cité en n'étant pas poursuivi en justice ${ }^{30}$, c'est surtout son caractère violent qui est pointé du doigt par Théomnestos dans la mesure où il risque de semer le chaos dans la cité en bafouant les lois naturelles et humaines ${ }^{31}$. Si l'injure de Théomnestos sert une possible manœuvre judiciaire, elle contribue plus encore à montrer que le client de Lysias est un citoyen dangereux pour les siens en raison de son incapacité à honorer une morale collective qui, 
située à la confluence entre la morale hoplitique et la philosophie platonicienne ou aristotélicienne ${ }^{32}$, tend à considérer l'homme accompli comme un être parvenant, pour le bien de la communauté civique, à résister à ses appétits physiques et à ses passions meurtrières ${ }^{33}$. En phase avec les préoccupations de son époque, l'attaque verbale de Théomnestos pourrait bien faire mouche auprès d'un jury dont la sensibilité à l'égard du parricide est aiguisée par le souvenir des pratiques criminelles propres à la stasis qui a déchiré Athènes à la fin $d u v^{e}$ siècle $^{34}$ mais aussi par les hommes de lettres qui, déstabilisés par les progrès de l'impiété filiale ${ }^{35}$ lors de la Guerre du Péloponnèse, n'ont de cesse de condamner les mauvais fill ${ }^{36}$ dont l'incapacité à modérer leurs passions et à réfréner leur égoïsme brutal pourrait saper les fondements de la société athénienne (Menu 2000 et Larran 2011, p. 95-115).

12 La réplique du client de Lysias répond en miroir aux attaques de Théomnestos. Motivée par des objectifs comparables, soutenue également par un arsenal de topoi civiques, elle inverse les rôles en retournant contre son adversaire ses propres armes. D'une plaidoirie à l'autre, les acteurs du procès changent de personnage et l'injure verbale change de camp. Aux habits du mauvais fils emporté par le déchaînement de ses passions meurtrières que lui imposait Théomnestos, le client de Lysias préfère le costume du plaideur tranquille ${ }^{37}$, inexpert en chicane et procédures judiciaires ${ }^{38}$, étranger aux paroles injurieuses et agressives ${ }^{39}$, mais qui a le devoir moral de répliquer aux insultes s'il ne veut se déshonorer, car seules les âmes esclaves acceptent de les endurer sans réagir ${ }^{40}$. Dans le discours du client de Lysias, Théomnestos est lui aussi appelé à changer de rôle. Au personnage contempteur des mœurs de son adversaire succède ainsi la figure du mauvais citoyen dont le langage intempérant porte la marque de ses défauts moraux : la colère, la lâcheté, l'irréflexion, l'insouciance et la paresse ${ }^{41} \ldots$ La violence condamnable passe elle aussi d'un camp à l'autre, du parricide à l'insulteur. La communauté est, à chaque fois, menacée. Si le parricide risque de saper la société en ses fondements, l'insulteur la souille en prononçant des mots interdits ${ }^{42}$. Le danger n'est pas seulement de nature religieuse. En attaquant le fils d'un stratège victime des Trente pour son dévouement à la cause du peuple $e^{43}$, Théomnestos pourrait prendre symboliquement le contrepied d'un processus de restauration démocratique engagée par Athènes aux lendemains de la Guerre du Péloponnèse. Religieuse, morale, politique, la menace est également d'ordre social, car elle envenime les relations humaines dans une cité qui cherche précisément à retrouver la concorde civile (homonoia) ${ }^{44}$. De l'injure naissent effectivement les inimitiés, la haine, les blessures et même les homicides ${ }^{45}$. C'est bien pour éviter une escalade de violence dangereuse pour la cité ${ }^{46}$ que la législation athénienne sanctionne les attaques verbales ( kakègorias dikai) ${ }^{47}$ et que Platon propose d'interdire les injures dans Les Lois ${ }^{48}$.

13 Le portrait de Théomnestos brossé par le client de Lysias est-il suffisamment accablant ? $\mathrm{Au}$ topos du mauvais citoyen, semeur de discorde, d'impiété et d'immoralisme, s'ajoute le topos du méchant orateur, comme le suggère l'offense exprimée par le plaideur face à la violence injurieuse de son adversaire ${ }^{49}$. L'attaque verbale de Théomnestos contrarie en effet les règles de la bienséance oratoire $\mathrm{du}_{\mathrm{IV}}^{\mathrm{e}}$ siècle qui imposent au citoyen une parole réfléchie, courtoise et modérée ${ }^{50}$ dans les conversations quotidiennes comme à la tribune 51 .

14 Une mise en perspective historique des propos tenus par Théomnestos semble nécessaire pour en cerner la portée culturelle. La Guerre du Péloponnèse sert une nouvelle fois de repoussoir. Les dérapages des démagogues athéniens qui ont sévi lors du conflit permettent effectivement aux Athéniens du $\mathrm{IV}^{\mathrm{e}}$ siècle d'opposer ${ }^{52}$ le modèle idéalisé de 
l'ancien orateur au maintien grave et solennel, à l'image de Solon, d'Aristide, de Thémistocle, de Miltiade ou de Périclès ${ }^{53}$, à son contre-modèle détestable bâti à partir du personnage de Cléon. La Constitution des Athéniens d'Aristote insiste précisément sur la rupture que le démagogue athénien, haï tant par Thucydide que par Aristophane pour ses gesticulations, la violence de ses propos et sa grossièreté $e^{54}$, a provoqué dans l'histoire de l'art oratoire ${ }^{55}$ :

Après la mort de Périclès, le chef des gens en vue fut Nicias, celui qui périt en Sicile ; celui du peuple fut Cléon, fils de Cléainétos, qui paraît avoir le plus corrompu le peuple par ses emportements et qui le premier cria à la tribune, y employa des injures et parla tout en se débraillant alors que les autres orateurs gardaient une attitude correcte ${ }^{56}$.

Régulièrement utilisé dans les discours des orateurs attiques, le topos du mauvais orateur pourrait encore s'appliquer à Théomnestos qui, comme Cléon, n'hésite pas à utiliser, dans ses discours, de violents propos nourris à la source de la colère pour servir ses intérêts personnels. Outrageante pour la partie adverse, l'attaque verbale risque également de tromper les jurés du procès qui pourraient, à l'instar des auditoires trop facilement séduits par les insultes des mauvais orateurs, des démagogues ou des sycophantes ${ }^{57}$, céder aux sirènes de l'injure calomnieuse de Théomnestos et oublier ainsi de respecter les lois établies et les serments qu'ils ont prêtés avant de rendre la justice ${ }^{58}$.

Armes au service des stratégies judiciaires, traits acérés dirigés contre les déviants ou bien marques infamantes des citoyens impies et immoraux, les injures et les interprétations qui en sont données sont lourdes de sens dans les discours des orateurs attiques. Dans le Contre Théomnestos, elles engagent pleinement les plaideurs dans leur rapport à la cité.

\section{De l'interprétation de l'injure à la démonstration de fidélité civique}

17 Artifices rhétoriques, finasseries opportunistes ou bien raisonnements subtils? Comment qualifier les arguments alimentant les interprétations contradictoires de l'attaque verbale de Théomnestos lors de son procès ? Dans une cité qui peine à se remettre de la Guerre du Péloponnèse dont la brutalité a modifié jusqu'au sens commun des termes en usage ${ }^{59}$, l'interprétation à donner aux mots se révèle de la plus grande importance, surtout lorsqu'elle conditionne la compréhension ${ }^{60}$ et l'application d'une législation archaïque au langage obscur ${ }^{61}$ qui est censée assurer, au début $d u \mathrm{IV}^{\mathrm{e}}$ siècle, la viabilité du régime démocratique athénien et le salut de la cité.

Pour Théomnestos, il faut s'en tenir à une interprétation littérale de la loi :

[Théomnestos] répétera devant vous ce qu'il a osé soutenir déjà devant l'arbitre, que ce n'est pas prononcer un des mots interdits de dire que quelqu'un a tué son père; que la loi ne défend pas cela, mais l'emploi du terme «meurtrier » ( androphonos) ${ }^{62}$.

19 Aussi spécieux et artificiel qu'il paraisse, l'argument ne relève sans doute pas d'une simple pirouette intellectuelle guidée par une mauvaise foi opportuniste. Pour preuves de la légitimité de la défense de Théomnestos, on retiendra la longueur de la plaidoirie de son adversaire qui multiplie les arguments pour tenter de convaincre les jurés ainsi que le caractère sacrilège des mots interdits dont l'énonciation constitue en elle-même un délit. Le point de vue de Théomnestos semble également conforme à la prise de position 
aristotélicienne quant à l'interprétation à donner aux lois de Solon rédigées dans une langue difficilement compréhensible pour les Athéniens de l'époque classique. La Constitution des Athéniens estime ainsi qu'il faut les comprendre en les replaçant dans l'ensemble de la constitution du législateur et non à la lumière d'une interprétation moderne :

Comme les lois n'étaient pas rédigées d'une façon claire et simple [...], il en résultait nécessairement beaucoup de contestations, et le tribunal décidait de toutes les affaires publiques et privées. Aussi certains croient-ils que c'est intentionnellement que Solon rédigea ses lois de façon obscure, afin de rendre le peuple maître des jugements. Cela n'est pas vraisemblable, et ce défaut provient de l'impossibilité d'arriver à la perfection avec une règle générale. Il n'est pas juste d'apprécier l'intention de Solon d'après ce qui se produit maintenant, mais il faut le faire d'après l'ensemble de sa constitution ${ }^{63}$.

Théomnestos n'est sans doute pas ainsi le seul au $\mathrm{IV}^{\mathrm{e}}$ siècle ${ }^{64}$ à préférer la lettre des textes de lois aux interprétations jurisprudentielles nouvelles qui auraient comme intention d'élargir le principe législatif, au risque de fausser le sens de la loi. L'occasion est sans doute trop belle pour qu'elle n'ait pas été exploitée par Théomnestos : le topos du mauvais citoyen artisan de ses propres lois, souvent condamné par les orateurs attiques ${ }^{65}$, s'offre à lui comme une arme utile pour déconsidérer un adversaire tenté par une interprétation extensive des lois.

La plaidoirie du client de Lysias répond point par point à celle de Théomnestos pour mieux retourner chacun de ses arguments. Contre l'accusation de forger des lois à son propre usage, il rappelle que Théomnestos a lui-même utilisé différemment la loi sur les aporrhèta afin de servir ses intérêts particuliers :

D'ailleurs, toi-même, tu as intenté l'action d'injures contre Lysithéos, qui avait dit que tu avais jeté ton bouclier; mais il n'est pas question de «jeter» dans la loi : la loi condamne à une amende de 500 drachmes celui qui dit « un tel a abandonné son bouclier ». N'est-il pas scandaleux que, lorsque tu as besoin d'obtenir réparation d'une injure, tu prennes la loi comme je le fais maintenant et qu'en revanche, lorsque tu injuries les autres au mépris des lois, tu prétendes ne pas être puni ? Estu donc assez habile pour pouvoir jouer des lois à ta fantaisie ${ }^{66}$ ?

Contre l'interprétation littérale des mots de la loi prônée par Théomnestos, le client de Lysias préfère retenir le sens des termes aporrhèta ${ }^{67}$ arguant du fait qu'un terme et une phrase peuvent désigner un même acte ${ }^{68}$, que des termes synonymes ne doivent pas être distingués les uns des autres ${ }^{69}$ et que des termes nouveaux peuvent désigner la même réalité que des termes anciens ${ }^{70}$. C'est bien contre des arguments de nature sophistique que le client de Lysias doit ici se battre. La défense de Théomnestos fondée sur une distinction jugée absurde ${ }^{71}$ entre le terme " meurtrier » (androphonos) et l'expression " tu as tué ton père» paraît en effet s'inscrire dans le prolongement de la théorie de «l'exactitude des noms» (orthotès tôn onomatôn) développée par les sophistes. Envisagée tout d'abord par Démocrite qui s'intéresse à la polysémie des noms ${ }^{72}$, approfondie par le père de l'éristique ${ }^{73}$, Protagoras, dont l'orthoépeia "justesse des noms " ${ }^{74}$ suggère de recourir au sens propre et non au sens figuré des noms afin de rendre fort l'argument faible par une utilisation opportune, dans le discours, des termes les plus clairs ${ }^{75}$, l'acribie devient une théorie très influente à la fin $d u v^{e}$ siècle ${ }^{76}$ grâce à Prodicos, qui meurt quelque temps avant le procès de Théomnestos. Convaincu que chaque terme exprime une seule chose contrairement à son usage courant $^{77}$, le sophiste de Céos entend distinguer les mots de sens voisin (diairesis tôn onomatôn) ${ }^{78}$ avec une telle exactitude qu'il en vient à refuser l'existence de parfaits synonymes ${ }^{79}$. Sa pensée est encore poursuivie par 
Antisthène le socratique qui établit la nécessité de "fixer " le sens propre de chaque terme. Si sa théorie du langage le conduit peut-être à imaginer qu'on ne peut pas parler de manière fausse car il n'est possible d'évoquer une chose que par son propre $\operatorname{logos}^{80}$, elle lui permet également de mener, vers 390 , des exégèses homériques ${ }^{81}$ au cours desquelles il se propose d'établir le sens exact du mot polutropos afin de montrer qu'il n'est pas péjoratif et que l'évaluation négative d'Ulysse à l'époque classique doit être révisée.

Que Théomnestos soit réellement influencé par les théories sur le langage de son temps ou qu'il les exploite sciemment, l'essentiel pour le client de Lysias est sans doute de le présenter comme un émule des sophistes. En distinguant les mots, en refusant les synonymies trop évidentes pour rendre fort ses arguments, en se livrant à une exégèse littérale d'une ancienne loi pour battre en brèche l'interprétation courante d'un de ses termes, Théomnestos agit finalement comme Protagoras, Prodicos ou Antisthène. Facilité par l'actualité intellectuelle d'Athènes au début $\mathrm{du}_{\mathrm{Iv}}^{\mathrm{e}}$ siècle $\mathrm{e}^{82}$, le rapprochement de Théomnestos avec de tels personnages pourrait bien contribuer à le décrédibiliser auprès de jurés populaires volontiers hostiles aux innovations sophistiques et habitués à se moquer, au théâtre, de leurs auteurs. Par les critiques adressées à son adversaire, le client de Lysias cherche ainsi sans doute à réactiver le topos du dangereux sophiste ${ }^{83}$, dont les origines remontent probablement aux Nuées d'Aristophane et qui sert encore de repoussoir dans la philosophie platonicienne ou aristotélicienne. Tel Phidippide qui, passé maître en rhétorique retorse grâce à Socrate, impose une nouvelle interprétation de la loi en jouant sur le nom du jour d'acquittement des dettes de son père pour ne pas les rembourser ${ }^{84}$, Théomnestos manipule les mots comme les lois sur les aporrhèta pour mieux les contourner ${ }^{85}$. Tel le Socrate d'Aristophane invitant Strepsiade à débattre sur le genre des mots, Théomnestos voit l'artificialité de ses raisonnements contrer par le bon sens commun ${ }^{86}$. Enfin, tels les sophistes mis face à leurs contradictions par le Socrate de Platon, Théomnestos est condamné pour ses distinctions sémantiques artificielles ${ }^{87}$ ou même encore pour l'utilisation immorale de son $\log _{0}{ }^{88}$.

Théomnestos, un sophiste? La menace est grave, la réaction du client de Lysias immédiate. Il en va en effet de son sort personnel comme de celui du jury. À l'image du public inactif des sophistes séduit par les querelles éristiques tournant à vide ${ }^{89}$, les jurés pourraient substituer à leur vigilance l'inertie intellectuelle en se laissant dominer par le plaisir d'écouter la vaine controverse sur les mots lancée par Théomnestos. Pour contrer le danger et réintégrer son adversaire dans le corps des bons citoyens, le client de Lysias accepte de troquer provisoirement son costume de plaideur contre celui d'éducateur :

Je pense, juges, que vous voyez tous que j'ai raison, et qu'il n'a même pas l'intelligence de comprendre le sens des mots. Je vais donc, en prenant d'autres lois, essayer de l'instruire (didaxai) là-dessus : voyons s'il fera son éducation (paideuthè) aujourd'hui du moins, à la tribune, et s'il s'abstiendra dorénavant de nous chercher noise. Lis-moi ces vieilles lois de Solon ${ }^{90}$.

L'éducation de Théomnestos doit ainsi passer par une interprétation de vieilles lois de Solon dont le sens et les valeurs sont appelées à traverser, immuables, les siècles et non à subir d'incessants changements, légitimés par le relativisme sophistique. L'éducation de Théomnestos ne peut ainsi se limiter à une simple éducation au langage mais doit être aussi considérée, plus fondamentalement, comme une éducation au temps de la cité. 


\section{L'injure entre deux conceptions du temps de la cité}

26

Si l'on se fie au client de Lysias, les désaccords qui rythment le procès de Théomnestos ne se réduisent pas à la seule interprétation textuelle de la loi sur les aporrhèta. Ils portent également sur le rapport à l'histoire de chacune des parties. Après avoir montré que les Athéniens continuent de se conformer à la pensée de Solon alors même qu'ils n'utilisent plus les termes archaïques de ses lois ${ }^{91}$, le client de Lysias conclut en effet :

Des exemples semblables ne manquent pas, juges : mais lui-même, s'il n'a pas la tête trop dure, il comprend bien, je pense, que les choses n'ont pas changé depuis le vieux temps, mais seulement, parfois, les termes en usage ${ }^{92}$. immuables, susceptibles de garder leur autorité à travers les vicissitudes de l'histoire. Reconnues comme supérieures ${ }^{93}$ et appelées de leurs vœux par les Athéniens à la fin du $v^{e}$ siècle et au début $\mathrm{du} \mathrm{IV}^{\mathrm{e}}$ siècle ${ }^{94}$, elles seules pourraient en effet garantir à la cité une stabilité salutaire. Les aspirations athéniennes s'expliquent encore une fois par les soubresauts de la Guerre du Péloponnèse, qui ont non seulement déstabilisé par deux fois le régime démocratique mais aussi profondément modifié la perception du temps. Comment est-il désormais possible de croire en la capacité d'une loi ancienne de traverser indemne les siècles si l'histoire est conçue comme une course imprévisible ${ }^{95}$ obéissant au hasard ${ }^{96}$ et scandée par de profonds changements ${ }^{97}$ ? Alors que, jusqu'à la Guerre du Péloponnèse, le temps était guidé par la justice (dikè) de façon régulière, on lui prête désormais comme moteur la physis humaine ${ }^{98}$ et ses caprices. De cette conception nouvelle de l'histoire résulte des querelles historiographiques ${ }^{99}$ sur l'interprétation à donner au passé d'Athènes et à sa tradition identitaire, alors qu'avant la Guerre du Péloponnèse une lecture commune des faits antérieurs confortait la cohésion de la cité ${ }^{100}$. La controverse sur l'ancrage dans le temps de l'injure androphonos qui oppose Théomnestos au client de Lysias semble symptomatique d'une cité en crise identitaire qui questionne son histoire.

ceptant que le «temps change les choses ", Théomnestos rend caduque l'application de la loi sur les aporrhèta et interdit de ce fait à la cité de s'appuyer sur de vieilles lois immuables. Son relativisme le condamne une nouvelle fois à se comporter en mauvais citoyen qui, attiré par les théories des sophistes ${ }^{101}$, refuse la permanence du temps civique. Considérer androphonos comme une insulte archaïque propre à son temps, c'est finalement interdire, selon le client de Lysias, aux valeurs nourrissant les anciennes lois de traverser les siècles et adopter, de la sorte, une démarche anticivique. Théomnestos pourrait bien agir en cela comme Phidippide qui prend, dans Les Nuées, le contrepied des lois anciennes sous prétexte qu'elles ne sont qu'une convention humaine appelée à être modifiée d'une époque à l'autre ${ }^{102}$. Dangereuse pour la cité, cette prise de position sophistique ${ }^{103}$ accepte l'inversion des valeurs et contribue à la dégradation générale des mœurs, vivement ressentie à partir de la Guerre du Péloponnèse ${ }^{104}$ et amèrement regrettée par les orateurs attiques du IV ${ }^{\mathrm{e}}$ siècle ${ }^{105}$.

Contre la position relativiste de Théomnestos, le client de Lysias défend la valeur intemporelle des choses, et en particulier de l'injure androphonos. De qui tient-il sa 
conception du temps? De Socrate qui, dans le Cratyle assure que les choses ont une nature fixe et que les termes qui les désignent peuvent être modifiés par l'usage et le temps ${ }^{106}$ ? Des partisans de «la loi naturelle qui a été fixée unique et identique pour tous les hommes et les animaux, [selon laquelle il faut] honorer ses parents $»^{107}$ ? Des bons citoyens convaincus du caractère intemporel de la cité athénienne? En refusant la relativité historique des valeurs, le client de Lysias fait sienne la conception de l'histoire à l'œuvre dans les oraisons funèbres ${ }^{108}$. Comme en témoigne celle composée par Lysias ${ }^{109}$, les bouleversements historiques n'ont pas de prise sur l'essence éternelle de la cité athénienne. Par delà les guerres civiles et les changements de régime politique, la cité prétend traverser indemne les époques et assurer la pérennité des valeurs fondamentales qui ont fait sa renommée dans les siècles passés ${ }^{110}$. C'est bien parce que les Athéniens sont d'une nature immuable, intrinsèquement bonne et démocrate, qu'ils peuvent, en se plaçant à l'écoute de la grandiose renommée de leurs ancêtres ${ }^{111}$, tenter de les imiter ${ }^{112}$ et ainsi bâtir un avenir glorieux pour leur cité. L'idée ne fait pas de doute pour Isocrate, car : « il est évident que la situation qui existait pour nous nos ancêtres se reproduira pour nous, puisque nécessairement de la même politique résultent toujours des actes semblables ou analogues ${ }^{113}$. Bien commun à la disposition de tous ${ }^{114}$, le passé athénien recèle autant d'actes de bravoure que de lois appelées à être suivies et respectées pour assurer la pérennité de la cité. En choisissant de reconnaître le caractère intemporel de la valeur de l'insulte "androphonos », le client de Lysias semble, une fois de plus, faire acte d'allégeance à la cité athénienne, acceptée dans ses lois, ses valeurs, ses mots ainsi que son temps idéalisé.

31 Théomnestos était-il coupable ? En l'absence de donnée sur l'issue du procès, il est bien difficile de savoir si les jurés l'ont tenu responsable du délit d'injure. Il faut s'en consoler en notant que l'affaire Théomnestos est autant un fait divers judiciaire qu'un moment civique. Injure impie, injure illégale, injure manipulée, injure cristallisant les angoisses athéniennes aux lendemains de la Guerre du Péloponnèse, injure prétexte aux démonstrations de fidélité civique, injure miroir des débats intellectuels et des controverses sophistiques, l'attaque verbale "tu as tué ton père » n'est pas seulement destinée au client de Lysias. Lu, repris ${ }^{115}$ et classé parmi les meilleurs discours des orateurs attiques dès la fin du IV siècle, le Contre Théomnestos s'offre, à l'instar des autres discours de logographes, comme un outil utile à la construction de la cité athénienne. Sa vocation pédagogique est multiple. Non content de rappeler à Théomnestos ses devoirs de citoyen, de lui apprendre à lire la loi et de lui donner une leçon sur le temps civique, le discours de Lysias a vocation à assurer l'éducation de l'ensemble des citoyens athéniens. $\mathrm{Au}$ service d'un procès appelé à être connu de tous ${ }^{116}$, le Contre Théomnestos impose aux jurés de se comporter de façon exemplaire car leur jugement déterminera le comportement de leurs contemporains. Punir un coupable au tribunal, ce n'est pas seulement condamner un particulier mais montrer, à tous, la voie à emprunter. Les verdicts rendus par l'Héliée sont autant de jalons susceptibles de conditionner l'avenir de la cité athénienne ${ }^{117}$ et les injures autant de sujets à débats nécessaires pour la guider dans la voie de la restauration démocratique. 


\section{BIBLIOGRAPHIE}

Andrews J. A. (1994), « Cleon's ethopoetics », CQ 44, p. 26-39.

Brancacci A. (2005) [1990], Antisthène, Le discours propre, Paris.

Bremmer J. (1991), «Walking, standing and sitting in ancient Greek culture », dans Bremmer J. et Roodenburg H. éd., A Cultural History of Gesture, Cambridge, p. 15-35.

Cambiano G. (1993), « Devenir homme », dans Vernant J.-P. dir., L’homme grec, Paris, p. 121-170.

Celato S. (1980-1981), « Homonoia e polis greca », CRDAC 11, p. 265-269.

Classen C. J. (1976), « The study of language amongst Socrates' contemporaries », dans

Classen C. J. éd., Sophistik, Darmstadt, p. 215-247.

Damet A. (2012), La septième porte. Les conflits familiaux dans l'Athènes classique, Paris.

Darbo-Peschanski C. (2007), L'historia, commencements grecs, Paris.

Demont P. (1990), La cité archaïque et classique et l'idéal de la tranquillité, Paris.

Hansen M. H. (1991), La démocratie athénienne à l'époque de Démosthène, Paris.

Hartog F. (1999), L'histoire d'Homère à Augustin, préfaces des historiens et textes sur l'histoire, Paris.

Hillgruber M. (1988), Die zehnte Rede des Lysias, Einleitung, Text und Kommentar mit einem Anhang über die Gesetzeinterpretationen bei den attischen Rednern, Berlin-New York.

Gagarin M. (2005), « La violence dans les plaidoyers attiques », dans Bertrand J.-M. dir., La violence dans les mondes grec et romain, Paris, p. 365-376.

Gernet L. (2001) [1917], Recherches sur le développement de la pensée juridique et morale en Grèce, Paris.

Glotz G. (1900), « Kakègorias dikè », dans Daremberg C. et Saglio E. éd., Dictionnaire des Antiquités grecques et romaines, Paris, p. 788-791.

Gotteland S. (2006), « La sirène et l'enchanteur : portraits croisés d'Eschine et de Démosthène sur la tribune ", REG 119-2, p. 588-608.

Guthrie W. K. C. (1976), Les sophistes, Paris.

Ismard P. (2013), L'événement Socrate, Paris.

Jacoby F. (1949), Atthis. The Local Chronicles of Athens, Oxford.

Larran F. (2011), Le bruit qui vole. Histoire de la rumeur et de la renommée en Grèce ancienne, Toulouse.

Loraux N. (1981), L'invention d'Athènes. Histoire de l'oraison funèbre dans la « cité classique », Paris.

Loraux N. (1997a), La cité divisée, l'oubli dans la mémoire d'Athènes, Paris.

Loraux N. (1997b), « La guerre dans la famille », Clio 5, p. 21-62.

Loraux N. (2005) [1986], « Thucydide et la sédition dans les mots », dans La tragédie d'Athènes. Le politique entre l'ombre et l'utopie, Paris, p. 81-107.

Marchiandi D. (2011), I periboli funerari nell'Attica classica, Athènes. 
Menu M. (2000), Jeunes et vieux chez Lysias. L'akolasie de la jeunesse au IV siècle avant J.-C., Rennes.

Momigliano A. (1969), « Prodico da Ceo e le dottrine sul linguaggio do Democrito ai Cinici », dans Quarto contributo alla storia degli studi classici e del mondo antico, Rome, p. 160-164.

Mossé C. (1979), « Comment s'élabore un mythe politique. Solon père fondateur de la démocratie athénienne », Annales ESC 34, p. 425-437.

Petre Z. (2000), «Le temps des ruptures », dans Constructions du temps dans le monde grec, Paris, p. 357-370.

Romilly de J. (1971), La loi dans la pensée grecque, Paris.

Romilly de J. (1986), « Les manies de Prodicos et la rigueur de la langue grecque », Museum Helveticum 43, p. 1-18.

Romilly de J. (1988), Les grands sophistes dans l'Athènes de Périclès, Paris.

Schutter de X. (1991), « Piété et impiété filiales en Grèce », Kernos 4, p. 219-243.

Todd S. C. (2007), A Commentary on Lysias Speeches, 1-11, Oxford.

Untersteiner M. (1993), Les sophistes, t. 2, Paris.

\section{NOTES}

1. Lysias, Contre Théomnestos (I), X, 1.

2. Lysias, Contre Théomnestos (I), X, 6.

3. Aristote, Problèmes, XXIX, 1. L'action d'injure n'est citée que par Lysias, Contre Théomnestos (I), X et XI, Isocrate, Contre Lochitès, XX, 3, Démosthène, Contre Midias, XXI, 32 et 81 et Contre Conon, LIV, 17-18. Voir ici Glotz 1900, p. 791.

4. Lysias, Contre Théomnestos (I), X, 2.

5. Aristote, Problèmes, XXIX, 14.

6. Plutarque, Vie de Solon, 21, 1-2.

7. Lysias, Contre Théomnestos (I), X, 1.

8. Sur ce point, voir Gernet 2001 (1917), p. 238-239.

9. Lysias, Contre Théomnestos (I), X, 12.

10. Sur les mots interdits dont le simple énoncé est en soi un crime : Lysias, Contre Théomnestos (I), X, 2-3 et 26 ; Contre Andocide, VI, 51. Sur le catalogue des mots interdits : Glotz 1900, p. 790 et Gernet 2001 (1917), p. 238-244.

11. Lysias, Contre Théomnestos (I), $\mathrm{X}, 12$.

12. Démosthène, Contre Euboulidès, LVII, 30-31.

13. Sur les débats historiographiques relatifs à cette question : Todd 2007, p. 625-626.

14. Voir ici Hillgruber 1988, p. 7 sq. qui confronte les différentes prises de position des spécialistes dans le débat historiographique. La question est notamment de savoir s'il faut faire remonter la loi sur les aporrhèta à Solon, à la fin de la période archaïque, au décret de Syrakosios de 415/4 (qui interdit aux poètes comiques de caricaturer les personnages politiques encore en vie) ou bien seulement au début du IV ${ }^{\mathrm{e}}$ siècle.

15. Gernet 2001 (1917), p. 240-244.

16. Fréquemment utilisé par Homère et Hésiode, le terme androphonos l'est beaucoup moins par les auteurs du v $\mathrm{v}^{\mathrm{e}}$ siècle (Eschyle, Les Sept contre Thèbes, 572 ; Pindare, Pythiques, IV, 252 et Euripide, Hécube, 1061). Au IV siècle, il revient plus régulièrement chez Lysias [Contre Théomnestos (I), X, 7], 
Isocrate (Panégyrique, IV, 157), Platon (Euthyphron, 4d et 9a ; Phédon, 114a ; Les Lois, 916c) et Aristote (Éthique à Nicomaque, 1107a12; Problèmes, 951b3).

17. Eschyle, Les Euménides, 209-210 (mètraloias) ; Aristophane, Les Nuées, 908-912 (patraloias).

18. Lysias, Contre Théomnestos (I), X, 8.

19. Notamment Andocide, Sur les Mystères, I, 100 ; Eschine, Contre Timarque, I, 165.

20. Par exemple Dinarque, Contre Démosthène, 15 et 97.

21. Plutarque, Vie de Solon, 21, 1-2.

22. Sur la complexité du procès de Socrate, sa portée symbolique et ses interprétations, voir Ismard 2013.

23. Lysias, Contre Théomnestos (I), X, 30.

24. Lysias, Contre Théomnestos (I), $\mathrm{X}, 22$.

25. Lysias, Contre Théomnestos (I), X, 3, 26, 28 et 31.

26. Par exemple Antiphon, Sur le meurtre d'Hérode, $\mathrm{V}, 11$.

27. Voir ainsi Antiphon, Sur le choreute, VI, 35-36.

28. Lysias, Contre Théomnestos (I), X, 1.

29. Eschine, Contre Timarque, I, 28.

30. Antiphon, Première Tétralogie, II, 1, 2-3.

31. Démosthène, Philippiques, IV, 40.

32. Sur l'émergence d'un nouveau modèle d'homme au IV siècle: Cambiano 1993, p. 160 et p. 166-167.

33. Lysias, Défense d'un anonyme, XXI, 19.

34. Les crimes familiaux et les guerres impies menées contre ses parents sont effectivement emblématiques des horreurs de la stasis provoquée par le régime des Trente, comme le suggère Lysias, Contre Ératosthène, XII, 92. Voir ici Loraux 1997b.

35. De Schutter 1991, p. 219-243. D'une façon plus générale, consulter Marchiandi 2011 et Damet 2012.

36. Après avoir suivi l'enseignement de Socrate et en prenant à rebours les principes du Raisonnement Juste (Aristophane, Les Nuées, 990-999), Phidippide critique ainsi, dans Les Nuées, le bien-fondé de l'obligation de respecter son père (Aristophane, Les Nuées, 1421-1426).

37. Sur le topos du plaideur tranquille : Demont 1990, p. 95 sq.

38. Lysias, Contre Théomnestos (I), X, 2.

39. Les plaideurs se présentent souvent comme des ennemis de l'insulte: Démosthène, Sur la couronne, XVIII, 264.

40. Aristote, Éthique à Nicomaque, IV, 5, 1226 a 7-8.

41. Lysias, Contre Théomnestos (I), X, 1, 11, 14-16, 29 et 30.

42. Gernet 2001 (1917), p. 238-244.

43. Lysias, Contre Théomnestos (I), X, 3 et 27.

44. Sur l'omniprésence de la notion d'homonoia dans les discours des orateurs attiques après la Guerre du Péloponnèse : Celato 1980-1981.

45. Par exemple Lysias, Sur le meurtre d'Ératosthène I, 45 et Contre Simon III, 43.

46. Sur la réprobation croissante de la violence dans les discours des orateurs au $\mathrm{IV}^{\mathrm{e}}$ siècle : Gagarin 2005, p. 368.

47. Démosthène, Contre Conon, LIV, 17-19.

48. Platon, Les Lois, XI, 934 d 5-935 a 2.

49. Lysias, Contre Théomnestos (I), X, 26.

50. Sur l'attitude de sôphrosunè attendue des citoyens dans leurs discours : voir Gotteland 2006, p. 588-608.

51. Démosthène, Sur la Couronne, XVIII, 256.

52. Sur l'attitude attendue d'un homme à l'époque classique : Bremmer 1995, p. 16-23 et p. 27-29. 
53. Voir ici Eschine, Contre Timarque, I, 25-26 ; Contre Ctésiphon, III, 2 ; Isocrate, Sur la paix, VIII, 75 ; Plutarque, Vie de Périclès, 5, 1.

54. Alors que Thucydide, III, 36, 6 le présente comme le « plus violent des citoyens ", Aristophane condamne ses vociférations agressives (Aristophane, Les Cavaliers, 256, 286-296, 626-629; Les Guêpes, 35-36, 536-537, 1034) et sa vulgarité (Aristophane, Les Cavaliers, 310).

55. Par exemple sur le style oratoire de Cléon : Andrews 1994.

56. Aristote, Constitution des Athéniens, 28, 3 (traduction G. Mathieu et B. Haussoulier).

57. Notamment Démosthène, Sur la couronne, XVIII, 3-4 ; Isocrate, Sur la paix, VIII, 123-124 ; Panathénaïque, XII, 135-137.

58. Lysias, Contre Théomnestos (I), X, 32.

59. Thucydide, III, 82, 2-5. L'idée se poursuit au IV siècle, comme le montrent Platon, La République, VIII, 560d-561a, Isocrate, Sur l'échange, XV, 283-284 et Aréopagitique, 20 pour lesquels le changement du sens des mots est un symptôme du dérèglement moral des individus. Voir ici Loraux 2005 (1986), p. 81-107.

60. Sur la difficulté des Athéniens à comprendre les termes utilisés dans les vieilles lois de Solon : Lysias, Contre Théomnestos (I), X, 16 sq.

61. Au lendemain de la restauration démocratique de 403, les Athéniens choisissent, par le décret de Tisaménos, de réviser les lois de Dracon et de Solon et de les afficher au Pœcile afin d'en assurer la compréhension et une large publicité (Andocide, Sur les Mystères, I, 82-83).

62. Lysias, Contre Théomnestos (I), X, 6.

63. Aristote, Constitution des Athéniens, $9,2$.

64. Qu'il s'agisse d'interpréter de vieilles lois archaïques ou des événements passés, Aristote, Théomnestos et Isocrate se méfient à chaque fois du pouvoir déformant des interprétations contemporaines. Isocrate, Panégyrique, IV, 8-9 rappelle notamment que le logos conditionne l'interprétation des événements passés et peut, d'un discours à l'autre, en modifier le sens.

65. Par exemple Antiphon, Sur le meurtre d'Hérode, V, 12-15.

66. Lysias, Contre Théomnestos (I), X, 12-13.

67. Lysias, Contre Théomnestos (I), X, 7.

68. Lysias, Contre Théomnestos (I), X, 8.

69. Lysias, Contre Théomnestos (I), X, 7.

70. Lysias, Contre Théomnestos (I), X, 16-20.

71. Lysias, Contre Théomnestos (I), X, 12.

72. Classen 1976, p. 241.

73. Diogène Laërce, IX, 52.

74. Platon, Phèdre, 267c et Classen 1976, p. 220.

75. Classen 1976, p. 222-226 et p. 230-231.

76. L'enseignement de Prodicos connaît, à son époque, un tel succès qu'il influence de façon probable Hérodote, Thucydide, Euripide, Xénophon, Antiphon et Socrate (notamment Romilly 1986).

77. Untersteiner 1993, p. 20-21.

78. Platon, Protagoras, 358a ; Charmide, 163d et Lachès, 197d. Sur la théorie du langage de Prodicos : Momigliano 1969, p. 160-164.

79. Prodicos établit souvent ainsi des distinctions sémantiques entre des paires de termes relevant de la psychologie morale : vouloir et désirer (Platon, Protagoras, 340a-b), être et devenir (Platon, Protagoras, 340b) ou encore travailler et fabriquer (Platon, Charmide, 163b-c).

80. Sur cette théorie et sa parenté antisthénienne : Guthrie 1976, p. 231.

81. Brancacci 2005, p. 43 sq.

82. On rappellera ainsi que Prodicos meurt vers 390, qu'Antisthène se livre à ses exégèses homériques à la même époque et que Platon pourrait avoir composé durant cette même période 
le Cratyle pour tenter de savoir si le nom des choses possède une justesse inhérente, naturelle ou s'il est un signe conventionnel.

83. Afin de discréditer Gorgias, Lysias utilise encore le topos du sophiste manipulateur de la langue dans un discours sans doute contemporain du procès contre Théomnestos : «Pour moi, je ne viens pas discourir sur de vains objets, ni disputer sur des mots : j'estime que cette besogne est bonne pour de frivoles sophistes qui ont grand besoin de gagner leur vie, mais que le devoir d'un bon citoyen, d'un homme de mérite, est d'éclairer les autres sur leurs intérêts essentiels » (Lysias, Discours Olympique, XXXIII, 3).

84. Aristophane, Les Nuées, 1178-1200.

85. Lysias, Contre Théomnestos (I), X, 12.

86. On mettra ainsi en parallèle les critiques du client de Lysias « le débat ne doit pas porter sur les mots, mais sur leur sens ; tout le monde sait que ceux qui ont tué sont meurtriers et que ceux qui sont meurtriers ont tué " et "Tu discutes sur les mots au lieu de considérer les choses auxquelles tout le monde applique les mots » [Lysias, Contre Théomnestos (I), X, 7 et 10] avec celle de Strepsiade dirigée contre Socrate: «Mais pourquoi me faut-il apprendre ce que nous savons tous? » (Aristophane, Les Nuées, 658-693, et notamment 690 ; traduction H. Van Daele).

87. Lysias, Contre Théomnestos (I), X, 9 reproche ainsi à Théomnestos de distinguer artificiellement « jeter » et « abandonner » son bouclier. Chez Platon, Socrate reproche à Prodicos, sans toutefois condamner systématiquement sa méthode, d'établir des distinctions sémantiques entre des termes qui désignent une seule et même chose (Platon, Ménon, 75e et Protagoras, $358 \mathrm{~d}$-e).

88. Le client de Lysias assure que Théomnestos est favorable à l'idée de relâcher un voleur car il n'est pas qualifié de "détrousseur » ou d'acquitter un coupable qui se dirait meurtrier sous prétexte que le serment de l'accusateur mentionne seulement qu'il a tué [Lysias, Contre Théomnestos (I), X, 10 et 12].

89. Lors de la Guerre du Péloponnèse, Cléon reproche déjà aux Athéniens, réunis à l'ecclèsia, leur goût pour les argumentations à vide et les prie de ne pas devenir « victimes du plaisir de l'oreille, des spectateurs assis pour contempler des sophistes et non des citoyens qui délibèrent sur les affaires de l'État » (Thucydide, III, 38, 7).

90. Lysias, Contre Théomnestos (I), X, 15.

91. Lysias, Contre Théomnestos (I), X, 16-19.

92. Lysias, Contre Théomnestos (I), X, 20.

93. Notamment Antiphon, Sur le meurtre d'Hérode, $\mathrm{V}, 14$.

94. Voir ici Romilly 1971, p. 217.

95. Telle est par exemple la conception de l'histoire à l'œuvre dans les Helléniques de Xénophon, comme le montre l'issue incompréhensible de la bataille de Mantinée (362) : «après ces faits, ce qui se passa fut le contraire de ce que tous les hommes pensèrent qu'il arriverait » (Xénophon, Helléniques, 7, 5, 26 ; traduction M. Casevitz). Sur l'interprétation de cette bataille par Xénophon, voir Hartog 1999, p. 91 et Darbo-Peschanski 2007, p. 257.

96. Chez Euripide, le temps préside ainsi à des combinaisons chronologiques qui obéissent au hasard, comme en témoignent les intrigues de ses tragédies, complexes et riches en retournements.

97. Isocrate, Aréopagitique, VII, 6-8 est ainsi profondément marqué par les changements de l'histoire qui ont notamment conduit, à partir de la Guerre du Péloponnèse, à la destruction des plus grandes puissances. La Constitution des Athéniens d'Aristote scande quant à elle l'histoire de la cité en onze changements brutaux (metabolai).

98. Darbo-Peschanski 2007, p. 231 sq. et 286 sq.

99. Sur les interprétations divergentes du passé dans les écrits fragmentaires des Atthides, voir Jacoby 1949.

100. Petre 2000, p. 364. 
101. Protagoras établit ainsi que "l'homme est la mesure de toute chose » et admet ainsi que le monde réel est pour chacun différent (Platon, Théétète, 152a).

102. Aristophane, Les Nuées, 1420-1424.

103. Sur la conception sophistique de la loi comme simple convention humaine : Romilly 1971, p. 109 et 169.

104. Sur la crise morale provoquée par la peste et les staseis de la Guerre du Péloponnèse : Thucydide, II, 53 et III, 82 sq.

105. Par exemple Lysias, Contre Épicratès, XXVII, 11 ; Isocrate, Aréopagitique, VII, 76 et Sur l'échange, XV, 159-160.

106. Notamment Platon, Cratyle, 421d et Guthrie 1976, p. 214-217.

107. Démosthène, Contre Aristogiton (I), XXV, 65.

108. Loraux 1981, p. 119 rappelle à ce sujet : «Pour penser le temps, la polis n'utilise pas d'autre norme qu'elle-même : elle est dans le temps et le temps se concentre en elle ; la figure de la cité, projetée dans le passé ou exaltée dans le présent, aura plus ou moins d'actualité mais restera toujours la référence. »

109. Lysias, Oraison funèbre, II et son étude par Loraux 1981, p. 123-125.

110. Sur l'idéalisation du passé dans les oraisons funèbres athéniennes: voir Loraux 1981, notamment p. 326 .

111. Lysias, Oraison funèbre, II, 3.

112. Lysias, Oraison funèbre, II, 61.

113. Isocrate, Aréopagitique, VII, 78 (traduction G. Mathieu).

114. Isocrate, Panégyrique, IV, 9.

115. On possède ainsi une autre version plus courte du Contre Théomnestos: Lysias, Contre Théomnestos (II), XI.

116. Les affaires judiciaires instruites par les divers magistrats étaient inscrites sur des tableaux publics : Aristote, Constitution des Athéniens, 59, 52, 1 et 53, 1 ; Isocrate, Sur l'échange, XV, 237-239. 117. Par exemple : Lysias, Contre Alcibiade, XIV, 4.

\section{RÉSUMÉS}

Un travail de recontextualisation historique semble nécessaire pour comprendre les enjeux du procès de Théomnestos datant de 384-383. Le plaignant, le client de Lysias, reproche à Théomnestos d'avoir prétendu, au cours d'un précédent procès, qu'il avait tué son propre père. Théomnestos aurait, à cette occasion, fait allusion à un mot injurieux interdit par la loi (aporrhèta ). Dans l'Athènes du début du IV siècle, le délit est grave : prononcer une telle injure, c'est faire montre d'incivisme et réintroduire le désordre dans une cité qui a précisément comme ambition d'oublier les maux de la Guerre du Péloponnèse pour mieux se reconstruire sur la base d'un passé idéalisé. Cherchant chacun à se présenter au tribunal comme un citoyen exemplaire, les deux plaideurs prennent position à l'égard des aporrhèta et se livrent alors à une sorte de dokimasie au cours de laquelle les jurés interrogent leur comportement, leur rapport au langage ainsi qu'à l'histoire de la cité.

An historical recontextualisation seems to be necessary to understand what is at stake in Theomnestos 384-383 trial. The plaintiff, Lysias'client, blames Theomnestos for having claimed, in a previous trial, that he had killed his own father. On that occasion, Theomnestos would have 
made allusion to an offensive word prohibited by the law (aporrhèta). In the early IVth Century Athens, the offence is a serious one: delivering such an abuse is showing uncivism and reintroducing disturbance in a city which aims to forget the evils of the Peloponnesian War in order to rebuild itself on the basis of an idealized past. Each one of them tries to appear in court as a model citizen. Both the plaintiff and the defendant take a stand about the aporrhèta and practise a kind of dokimasie: the members of the jury interrogate them about their behaviour, their relation to language and to the History of the city.

INDEX

Mots-clés : injure, Lysias, sophiste, aporrhèta, tribunal

Keywords : offence, Lysias, sophist, aporrhèta, trial

\section{AUTEUR}

\section{FRANCIS LARRAN}

Lycée Martin Luther King, Bussy-Saint-Georges 